\title{
Ist das gesetzliche Hautkrebsscreening sinnvoll?
}

\section{Hier steht eine Anzeige.}

_ Seit Juli 2008 wird das Hautkrebsscreening von den Krankenkassen bezahlt. Alle zwei Jahre steht es gesetzlich Versicherten ab dem 35. Lebensjahr zu. Inwieweit das Screening die Erwartungen erfüllen konnte, erläuterte Prof. Claus Garbe, Tübingen. Hintergrund für das Screening war "ein potenzieller Nutzen durch die Vorverlegung des Diagnosezeitpunkts", so der Gemeinsame Bundesausschuss (G-BA) im Jahr 2008. Von der Diagnose in früheren Stadien versprach man sich eine schonendere, erfolgreichere Therapie sowie Kostenersparnis. Laut dem Barmer GEK "Arztreport 2014" leiden derzeit 1,5 Millionen Deutsche an Hautkrebs. „Extrapolieren wir diese Daten, wird sich die Inzidenz bis 2030 verdoppeln", sagte Garbe. Daher sei Frage nach dem Bedarf eines Screenings mit einem „uneingeschränkten Ja" zu beantworten. Zumal die diagnostischen Algorithmen für den Hautkrebsnachweis vorhanden seien. Die Erwartungen des G-BA nach schonenderer und kostengünstigeren Therapie bei Früherkennung hätten sich erfüllt. Allerdings hat das Screening die Mortalität - entgegen früherer Annahmen - bisher nicht gesenkt. So meldete das Robert Koch-Institut 2.030 Todesfälle wegen Hautkrebs im Jahr 1998, 2.500 in 2008 und 3.042 in 2013. „Das lässt darauf schließen, dass wir mit dem Hautkrebsscreening offensichtlich keinen Einfluss auf die Melanommortalität ausüben", so Garbe.

Als Grund für den Misserfolg hinsichtlich der Mortalität nannte Garbe quantitative Ursachen - die Teilnahme am Screening liegt nur bei etwa 34\%. Dies ergab eine FORSA-Befragung [Augustin M et al. JDDG. 2012;10:42-50]. Die Umfrage zeigte auch, dass sich etwa die Hälfte der gesetzlich Versicherten Sorgen wegen einer möglichen Hautkrebserkrankung machen. Über ihren Anspruch für ein Screening sind aber nur 50\% informiert.

Ein weiterer Grund für die Mortalitätssteigerung liegt laut Garbe an der diagnostischen Treffsicherheit. Über ihre Qualität ist wenig bekannt, sie sei bei Allgemeinärzten eventuell verbesserungswürdig. „Aus meiner Sicht bleibt die Verringerung der Mortalitätsrate ein wichtiges Ziel“", betonte Garbe. Er forderte die Qualifizierung von Allgemeinärzten für das Screening, die Einbeziehung weiterer Fachgruppen, sowie eine adäquate Vergütung für die Dermatoskopie. Zudem sollten DDG und BVDD die weitere Entwicklung des Screenings aktiv übernehmen. Dr.Marion Hofmann-Aßmus

Garbe C. Gesetzliches Hautkrebsscreening Sinn oder Unsinn?

\section{Onkologika: Kutane Nebenwirkungen managen}

- Kutane Nebenwirkungen treten unter Checkpointinhibitoren häufig auf, erreichen aber selten höhere Schweregrade. Vor allem Melanompatienten sind betroffen. „Vitiligo sehen wir bei etwa $10 \%$ der Melanompatienten unter Immuntherapie", sagte Prof. Ralf Gutzmer, Hannover. Vitiligo unter AntiPD-1-Therapie ist mit besserem Ansprechen assoziiert [Hua C et al. JAMA Dermatol. 2016; 52:45-51]. Zu beachten sei, dass diese Patienten weitere Autoimmunerkrankungen entwickeln können, etwa Typ-1-Diabetes. Beim Rash handelt es sich meist um makulopapulöse Exantheme, die mit topischen Steroiden therapierbar sind. Schwerere Fälle machen systemische Steroide und eventuell eine Therapiepause erforderlich. Therapiebegleitender Juckreiz ist laut Gutzmer häufig mild und lässt sich gut mit rückfettender Hautpflege, Antihistaminika und topischen Steroiden bei Ekzematisierung therapieren.

In Einzelfällen treten unter Anti-PD-1-Therapie entzündliche Hauterkrankungen auf, etwa Psoriasis (evtl. mit Nagelbeteiligung und Psoriasis-Arthritis), bullöse Dermatosen (z.B. bullöses Pemphigoid) oder Mundschleimhautveränderungen (z. B. Lichen ruber mucosae). Wie Gutzmer erklärte, bessern sich diese Nebenwirkungen unter systemischen Steroiden; eine Therapieunterbrechung ist ratsam. Da sich eine vorbestehende Psoriasis oder Psoriasis-Arthritis unter einer Anti-PD-1-Therapie verschlechtern kann, ist eine sorgfältige Nutzen-Risiko-Analyse erforderlich.

Dr. Marion Hofmann-Aßmus

Gutzmer R. Nebenwirkungsmanagement der modernen Onkologika 